\title{
Pseudo-Differential Operators on Manifolds with a Singular Boundary
}

\author{
Vladimir B. Vasilyev
}

\begin{abstract}
The aim of this work is to describe new interesting examples of nonsmooth manifolds and elliptic pseudo-differential operators acting in functional spaces on such manifolds. Fredholm properties for these operators are studied by factorization methods, and these are based on several complex variables.
\end{abstract}

Keywords Pseudo-differential operator • Local representative • Bochner operator • Wave factorization

Mathematics Subject Classification (2010) Primary 47G30; Secondary 32A07

\section{Introduction}

In 1990s the author has started to develop a new approach to pseudo-differential equations and boundary value problems on smooth manifolds with a non-smooth boundary. This approach takes its origin from Vishik-Eskin's theory of boundary value problems for manifolds with a smooth boundary [3]. The author has extended a factorization idea to non-smooth situation to apply it for describing Fredholm conditions for elliptic pseudo-differential equations and boundary value problems on manifolds with a non-smooth boundary. This studying was completed in general for a two-dimensional case [11] but last years the author has found new interesting constructions for a multidimensional case also [12-15]. A special case is the author's paper [10] in which it was done for Calderon-Zygmund operators only.

A basic problem is to describe Fredholm conditions for the equation

$$
(A u)(x)=v(x), \quad x \in M,
$$

\footnotetext{
V.B. Vasilyev $(\bowtie)$

Chair of Differential Equations, Belgorod National Research University, Studencheskaya 14/1, Belgorod 308007, Russia

e-mail:vbv57@inbox.ru
} 
where $M$ is a compact manifold with a boundary (non-smooth as a rule), $A$ is a pseudo-differential operator acting in certain functional spaces on $M$. We use Sobolev-Slobodetskii spaces $H^{s}(M), s \in \mathbb{R}$, for studying these properties [11]. Such spaces are introduced locally using a partition of identity [3].

Some words on other approaches to the problem and related topics [2, 4-8]. Richard Melrose's programme was declared in ICM-90 [5], and it is devoted to algebraical and topological aspects of the problem, this way is reserved by papers $[6,7]$. Other kind of papers was written by analysts. So, B.-W. Schulze and his group (see for example [2]) deals with general pseudo-differential operators on manifolds with cones and wedges.

"In all papers the conical domain (see Fig. 1 below) is treated as the direct product of a circle and a half-axis, then they apply the Mellin transform on half-axis, and the initial problem is reduced to a problem in a domain with a smooth boundary with operator-valued symbol. That follows further it is like the generalization of wellknown results on operator symbol case. Of course, the my approach is generalization also, but it is a generalization on dimension space, and the principal difference is that I don't divide the cone, and it's treated as an emergent thing" [14]. The last is related also to papers V. Mazya, B. Plamenevskii and others mentioned in [14].

Another kind of "analytical" papers deals with very "simple" operators and boundary value problems on manifolds with very "bad" boundary (see for example [4]). The main aim for them is developing the classical Fredholm theory as far as it is possible using potential theory.

Fig. 1 Simplest manifold with a non-smooth boundary

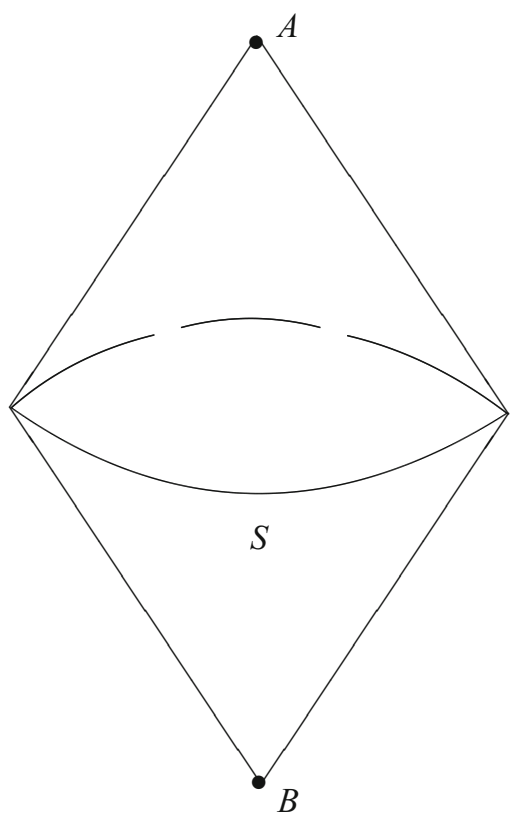


Finally recent paper [8] contains a certain new generalizations for statements of boundary value problems without smoothness requirement on boundary of a domain.

...we develop the global symbolic calculus of pseudo-differential operators generated by a boundary value problem for a given (not necessarily self-adjoint or elliptic) differential operator. For this, we also establish elements of a non-self-adjoint distribution theory and the corresponding biorthogonal Fourier analysis. There are no assumptions on the regularity of the boundary which is allowed to have arbitrary singularities. We give applications of the developed analysis to obtain a priori estimates for solutions of boundary value problems that are elliptic within the constructed calculus. [8]

\section{Non-Smooth Manifolds and Local Representatives}

Let it will not be a strange thing but in this section we'll define the declared manifold by operators which live on this manifold.

\subsection{Pseudo-differential Operators}

The main object of the paper is a linear bounded operator $A: H^{s_{1}}(M) \rightarrow H^{s_{2}}(M)$ which is called a pseudo-differential operator under following assumptions. We'll suppose that operator $A$ is composed by a certain operator-function $A\left(x_{0}\right), x_{0} \in \bar{M}$, so that for arbitrary smooth functions $\varphi, \psi$ on $M$ with supports concentrated in small neighborhoods $U, V(U \subset V)$ of $x$ the following representation for the operator $A$

$$
\varphi \cdot A \cdot \psi=\hat{\varphi} \cdot\left(A_{x_{0}}+T_{x_{0}}\right) \hat{\psi},
$$

holds, where $A_{x_{0}}$ is an operator defined by formula

$$
\left(A_{x_{0}} u\right)(x)=\int_{D} \int_{\mathbb{R}^{m}} e^{i \xi \cdot(x-y)} \tilde{A}\left(\eta^{-1}\left(x_{0}\right), \xi\right) \tilde{u}(\xi) d \xi d y, \quad x \in D
$$

$\eta: V \rightarrow D \subset \mathbb{R}^{m}$ is a local diffeomorphism, $\tilde{A}(x, \xi)$ is a certain function defined on $V \times \mathbb{R}^{m}, \hat{\varphi}=\varphi \circ \eta^{-1}, \hat{\psi}=\psi \circ \eta^{-1}, T_{x_{0}}: H^{s_{1}}\left(\mathbb{R}^{m}\right) \rightarrow H^{s_{2}}\left(\mathbb{R}^{m}\right)$ is a compact operator, $\tilde{u}(\xi)$ denotes the Fourier transform in $m$-dimensional space

$$
\tilde{u}(\xi)=\int_{\mathbb{R}^{m}} e^{i x \cdot \xi} u(x) d x
$$

$\forall u \in S\left(\mathbb{R}^{m}\right)$ (Schwartz class of infinitely differentiable rapidly decreasing functions at infinity). 
Remark 2.1 Generally speaking the domain $D$ depends on the point $x_{0}$ but we'll see below in a lot of cases it isn't essential.

Definition 2.2 The operator $A_{x_{0}}: H^{s_{1}}(D) \rightarrow H^{s_{2}}(D)$ is called a local representative of the operator $A: H^{s_{1}}(M) \rightarrow H^{s_{2}}(M)$ at the point $x_{0} \in \bar{M}$, and domain $D$ is called a canonical domain.

A structure of the set $D$ can be different in dependence on a placement of the point $x_{0}$. For inner points $\stackrel{\circ}{M}$ we have $D=\mathbb{R}^{m}$, for points of smoothness on $\partial M$ we have $D=\mathbb{R}_{+}^{m}$, for conical points $D=C_{+}^{a}$ and so on. One has painted a simple example of a non-smooth manifold $M$ on the Fig. 1 . There are distinct types of local representatives in dependence on a point kind. These local representatives are defined by different formulas for inner points $\stackrel{\circ}{M}$, for points of smoothness on $\partial M$, for points on $S$ which is a smooth edge of a wedge, and separately for conical points $A$ and $B$. Below we'll describe these local representatives and methods for their studying.

Definition 2.3 We say that a manifold $M$ has a non-smooth boundary if there is at least one local representative of a pseudo-differential operator for which $D \neq$ $\mathbb{R}^{m}, \mathbb{R}_{+}^{m}$, and the function $\sigma\left(x_{0}, \xi\right)=\tilde{A}\left(\eta^{-1}\left(x_{0}\right), \xi\right)$ defined on $\bar{M} \times \mathbb{R}^{m}$ is called a local symbol of a pseudo-differential operator $A$ at the point $x_{0}$.

\subsection{Canonical Domains: A Half-Space, a Cone, a Wedge}

Since the definition of a pseudo-differential operator is a local and we use the "freezing coefficients principle" or, in other words, "local principle" then we'll omit a pole $x_{0}$ in a symbol of a pseudo-differential operator. Thus we have following types of local operators related to an initial pseudo-differential operator $A$. First it is the operator

$$
u(x) \longmapsto \int_{\mathbb{R}^{m}} \int_{\mathbb{R}^{m}} \tilde{A}(\cdot, \xi) u(y) e^{i(x-y) \cdot \xi} d \xi d y, \quad x \in \mathbb{R}^{m}
$$

for a point $x_{0} \in \stackrel{\circ}{M}$.

If $x_{0} \in \partial M$ and $x_{0}$ is a smoothness point then we need another formula

$$
u(x) \longmapsto \int_{\mathbb{R}_{+}^{m}} \int_{\mathbb{R}^{m}} \tilde{A}(\cdot, \xi) u(y) e^{i(x-y) \cdot \xi} d \xi d y, \quad x \in \mathbb{R}_{+}^{m}
$$

For invertibility of such an operator with symbol $\tilde{A}(\cdot, \xi)$ not depending on a spatial variable $x_{0}$ one can apply the theory of the classical Riemann boundary 
value problem for upper and lower complex half-planes with a parameter $\xi^{\prime}=$ $\left(\xi_{1}, \ldots, \xi_{m-1}\right)$. This step was systematically studied in the book [3]. But if the boundary $\partial M$ has at least one conical point, this approach is not effective.

A conical point $x_{0}$ at the boundary is such a point for which its neighborhood is diffeomorphic to the cone $C_{+}^{a}=\left\{x \in \mathbb{R}^{m}: x_{m}>a\left|x^{\prime}\right|, x^{\prime}=\left(x_{1}, \ldots, x_{m-1}\right), a>\right.$ $0\}$, hence the local definition for pseudo-differential operator near the conical point is the following

$$
u(x) \longmapsto \int_{C_{+}^{a}} \int_{\mathbb{R}^{m}} \tilde{A}(\cdot, \xi) u(y) e^{i(x-y) \cdot \xi} d \xi d y, \quad x \in C_{+}^{a}
$$

A $k$-wedge point $x_{0}$ at the boundary is such a point for which its neighborhood is diffeomorphic to the wedge $W_{+}^{a_{k}, k}=\left\{x \in \mathbb{R}^{m}: x=\left(x^{\prime \prime}, x^{\prime}, x_{m}\right), x^{\prime \prime}=\right.$ $\left.\left(x_{1}, \cdots, x_{k}\right), x^{\prime}=\left(x_{k+1}, \cdots, x_{m-1}\right), x_{m}>a_{k}\left|x^{\prime}\right|, a_{k}>0\right\}$. In other words $W_{+}^{a_{k}, k}=\mathbb{R}^{k} \times C_{+}^{a_{k}}$, where $C_{+}^{a_{k}}$ is a cone in $\mathbb{R}^{m-k}$. Hence the local definition for pseudo-differential operator near the $k$-wedge point is the following

$$
u(x) \longmapsto \int_{W_{+}^{a_{k}, k}} \int_{\mathbb{R}^{m}} \tilde{A}(\cdot, \xi) u(y) e^{i(x-y) \cdot \xi} d \xi d y, \quad x \in W_{+}^{a}
$$

To study an invertibility property for the operator (2.3), (2.4) the author has introduced the concept of wave factorization for an elliptic symbol near a singular boundary point $[10,11]$ and using this property has described Fredholm properties for an equation with the operator $(2.3),(2.4)$.

\subsection{Class of Symbols}

To describe invertibility conditions for operators (2.1)-(2.3) we need to fix a class of local symbols under consideration.

Definition 2.4 A local symbol belongs to the class $S_{\alpha}$ if it satisfies the following condition

$$
\left|\sigma\left(x_{0}, \xi\right)\right| \sim(1+|\xi|)^{\alpha}, \quad \forall x_{0} \in \bar{M}, \quad \xi \in \mathbb{R}^{m}
$$

The number $\alpha$ is called an order of a pseudo-differential operator.

Such symbols and corresponding operators we call elliptic ones.

According to [3] such operators with local symbols from $S_{\alpha}$ are linear bounded operators acting from $H^{s}(D)$ to $H^{s}(D)$, and everywhere below we consider only symbols from the class $S_{\alpha}$. 


\subsection{Main Theorem}

Theorem 2.5 An elliptic pseudo-differential operator $A: H^{s}(M) \rightarrow H^{s-\alpha}(M)$ with continuous local symbol has a Fredholm property iff all local representatives $A_{x_{0}}$ : $H^{s}(D) \rightarrow H^{s-\alpha}(D)$ are invertible.

Proof Since the definition of a pseudo-differential operator given above assume that an operator is defined locally then a local principle [9] implies this assertion.

\subsection{Wave Factorization and Invertibility of Local Operators}

We'll give the definition of a wave factorization with respect to a $k$-dimensional wedge because a cone is a particular case of a wedge $W_{+}^{a_{0}, 0}=C_{+}^{a_{0}}$.

Let ${ }^{*}+\underset{+}{a}=\left\{x \in \mathbb{R}^{m}: a x_{m}>\left|x^{\prime}\right|\right\}$ be a conjugate cone, and $T\left(\stackrel{*}{C_{+}^{a}}\right)$ be a radial tube domain over the cone $C_{+}^{*}[1,11,16]$, it is a subset of $\mathbb{C}^{m}$ of the following type $T\left(\stackrel{*}{C_{+}^{a}}\right)=\mathbb{R}^{m}+i \stackrel{*}{C_{+}^{a}}$.

Definition 2.6 Wave factorization of a local elliptic symbol $\sigma\left(x_{0}, \xi\right)$ with respect to the wedge $W_{+}^{a_{k}, k}$ is called its representation in the form

$$
\sigma\left(x_{0}, \xi\right)=\sigma_{\neq}\left(x_{0}, \xi\right) \cdot \sigma_{=}\left(x_{0}, \xi\right)
$$

where factors admit analytic continuation into radial tube domains $T\left( \pm \stackrel{*}{a_{k}}\right)$ over cones $\pm C_{+}^{*} a_{k}$ for almost all $\xi^{\prime \prime}=\left(\xi_{1}, \cdots, \xi_{k}\right)$ with estimates

$$
\begin{gathered}
\left|\sigma_{\neq}^{ \pm 1}\left(x_{0}, \xi+i \tau\right)\right| \leq c_{1}(1+|\xi|+|\tau|)^{ \pm \mathfrak{x}_{k}}, \\
\left|\sigma_{=}^{ \pm 1}\left(x_{0}, \xi-i \tau\right)\right| \leq c_{2}(1+|\xi|+|\tau|)^{ \pm\left(\alpha-\mathfrak{x}_{k}\right)},
\end{gathered}
$$

where $c_{1}, c_{2}$ are constants.

The number $\mathfrak{x}_{k}$ is called an index of the wave factorization.

The following theorem in general was proved in [11]. There are some examples of elliptic symbols admitting the wave factorization.

Let us denote by $S_{k}$ a smooth sub-manifold of $M$ consisting of $k$-wedge points.

Theorem 2.7 If the elliptic local symbol $\sigma\left(x_{0}, \xi\right)$ admits wave factorization with respect to the wedge $W_{+}^{a, k}$ for all $x_{0} \in S_{k}$ and $|æ-s|<1 / 2$ then all local representatives $A_{x_{0}}, x_{0} \in S_{k}$, are invertible. 


\subsection{The Bochner Operator}

Let $S\left(\mathbb{R}^{m}\right)$ be the Schwartz space of infinitely differentiable rapidly decreasing at infinity functions. Let us define the following function

$$
B(z)=\int_{C_{+}^{a}} e^{i y \cdot z} d y, \quad z=x+i \tau \in T\left(C_{+}^{a}\right)
$$

and introduce the following

Definition 2.8 A Bochner operator is called the following linear operator

$$
(B u)(x)=\lim _{\tau \rightarrow 0} \int_{\mathbb{R}^{m}} B(z-y) u(y) d y,
$$

where $\tau \rightarrow 0$ along arbitrary non-tangential way, $\tau \in C_{+}^{*}[1,16]$.

Remark 2.9 For this case $C_{+}^{a}$ the Bochner kernel can be calculated exactly $[10,16]$

$$
B(z)=\frac{a \Gamma(m / 2)}{2 \pi^{\frac{m+2}{2}}} \frac{1}{\left(z^{\prime 2}-a^{2} z_{m}^{2}\right)^{m / 2}}, \quad z^{\prime}=\left(z_{1}, \cdots, z_{m-1}\right),
$$

where $\Gamma$ is Euler $\Gamma$-function, but all above is valid for arbitrary sharp convex cone in $\mathbb{R}^{m}$ although we don't know an explicit form of the kernel $B(z)$.

Proposition 2.10 The operator $B: L_{2}\left(\mathbb{R}^{m}\right) \rightarrow L_{2}\left(\mathbb{R}^{m}\right)$ is a linear bounded operator.

Proof It follows from the fact that the operator $B$ is Fourier image of a multiplication operator on an indicator of the cone $C_{+}^{a}$.

Remark 2.11 It is easy to prove that $B: H^{s}\left(\mathbb{R}^{m}\right) \rightarrow H^{s}\left(\mathbb{R}^{m}\right)$ is also linear bounded operator for $|s|<1 / 2$.

Let us note that the Bochner operator plays an important role and permits to construct an inverse operator for local operators (2.3), (2.4) [10,11].

\section{Hidden Parameters}

Everywhere above we have assumed that following parameters are constants. 


\subsection{Order of an Operator}

First an order of an pseudo-differential operator can vary from a point to a point. Simple example is an elliptic local symbol of following kind

$$
\sigma\left(x_{0}, \xi\right)=\left(1+|\xi|^{2}\right)^{\alpha\left(x_{0}\right)} .
$$

So there is the following

Problem 3.1 What one can say on boundedness and invertibility of such a pseudodifferential operator in Sobolev-Slobodetskii spaces $H^{s}(M)$ ?

\subsection{Index of Wave factorization}

Since index of factorization (according to Vishik-Eskin theory) determines a quantity of boundary conditions and index of wave factorization also it is very interesting situation when such indices vary from a point to a point. Thus the following question arises.

Problem 3.2 Is it possible the situation when one needs different quantity of boundary conditions on distinct parts of a boundary?

\subsection{A Variable Size of a Cone}

Here we consider a case when size of a cone varies from a point to a point. It means that size of a canonical cone $C_{+}^{a}$ can vary, in other words we need to consider a cone of a variable size $C_{+}^{a\left(x_{0}\right)}$. According to the definition of the operator $B$ one can construct the following operator

$$
\left(B_{v a r} u\right)(x)=\frac{a \Gamma(m / 2)}{2 \pi^{\frac{m+2}{2}}} \lim _{\tau \rightarrow 0+} \int_{\mathbb{R}^{m}} \frac{u(y) d y}{\left(\left(x^{\prime}-y^{\prime}\right)^{2}-a^{2}(x)\left(x_{m}-y_{m}+i \tau\right)^{2}\right)^{m / 2}} .
$$

Problem 3.3 What one can say on boundedness of the operator $B_{v a r}$ in SobolevSlobodetskii spaces $H^{s}\left(\mathbb{R}^{m}\right)$ ?

\section{A Wedge with a Variable Size}

Here we'll consider more complicated manifold $M$ with so-called $k$-wedges with a variable size. To study such singularities one can apply the developed technique for describing sufficient invertibility conditions of local operators. 
Definition 4.1 $k$-wedge of a variable size $W_{+}^{a_{k}\left(x_{0}\right), k}$ is a smooth sub-manifold $S_{k} \subset$ $M$ consisting of points $x_{0}$ in which a local representative of an operator $A$ has the form

$$
u(x) \longmapsto \int_{W_{+}^{a_{k}\left(x_{0}\right), k}} \int_{\mathbb{R}^{m}} \tilde{A}(\cdot, \xi) u(y) e^{i(x-y) \cdot \xi} d \xi d y, \quad x \in W_{+}^{a_{k}\left(x_{0}\right), k},
$$

where the function $a\left(x_{0}\right)$ is defined on $S_{k}$, is continuous, takes its values on an interval $\left(b_{k}, c_{k}\right)$ and has finite limits in points $b_{k}, c_{k}$.

\subsection{Refined Theorem and Sufficient Conditions}

Let a manifold $M$ be such that its boundary $\partial M$ includes a smooth part and smooth sub-manifolds $S_{k}$ which are $k$-wedges, $k=0, \cdots, m-2$. A sub-manifold $S_{m-1}$ is a closure of a smooth part of a boundary $\partial M$. For this piece of a boundary one can use Vishik-Eskin theory [3].

In this section we'll add to local representatives the operator (4.1) and formulate the following

Theorem 4.2 Let A be a pseudo-differential operator with continuous elliptic local symbol $\sigma\left(x_{0}, \xi\right)$. The operator $A: H^{s}(M) \rightarrow H^{s-\alpha}(M)$ has a Fredholm property iff all local representatives $A_{x_{0}}: H^{s}(D) \rightarrow H^{s-\alpha}(D)$ are invertible. If the local symbol admits the wave factorization with respect to $k$-wedge points $x_{0} \in S_{k},\left|\mathfrak{x}_{k}-s\right|<$ $1 / 2, k=0, \cdots, m-2$, then all such local representatives $A_{x_{0}}: H^{s}(D) \rightarrow H^{s-\alpha}(D)$ are invertible.

\subsection{From a Half-Space to a Half-Line: Degenerating Wedge}

If $b_{1}=0, c_{1}=+\infty$ we have the wedge on Fig. 2. For $b_{1}=0$ we obtain a plane, and for $c_{1}=+\infty$ we obtain a half-line. The author has made some attempts to describe such local representatives of an operator $A[13,14]$ but it is not clear up to now how one can work with such singularities.

\section{Exotic Singularities}

There are a lot of possibilities to construct another types of singularities combining mentioned above cones, wedges and their modifications. Some variants were presented in [12]. 


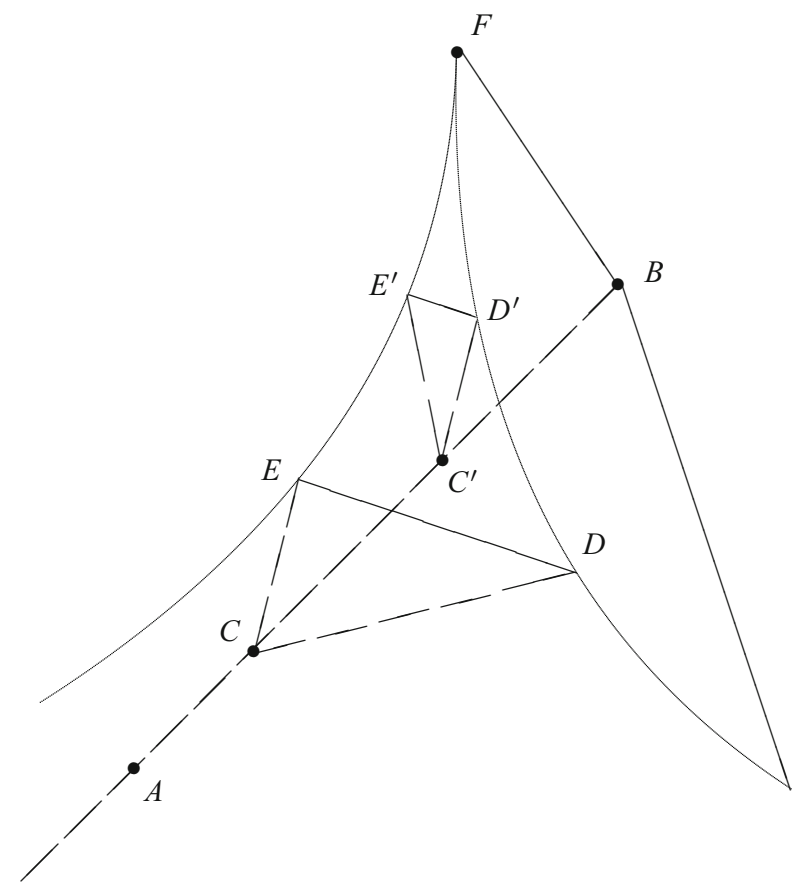

Fig. 2 A wedge of a variable size

\section{Conclusion}

The author hopes these considerations will help to enlarge a set of admissible manifolds with singular boundaries and to get answers to some formulated questions. It seems for all existing theories of boundary value problems for elliptic pseudo-differential equations on manifolds with singular boundaries one needs an invertibility of local representatives. Finding effective necessary and sufficient conditions for this property is a very hard problem, and any result in this direction will be a great achievement.

Acknowledgements This work was supported by the State contract of the Russian Ministry of Education and Science (contract No 1.7311.2017/B).

\section{References}

1. S. Bochner, W.T. Martin, Several Complex Variables (Princeton University Press, Princeton, 1948)

2. S. Coriasco, B.-W. Schulze, Edge problems on configurations with model cones of different dimensions. Osaka J. Math. 43, 63-102 (2006) 
3. G. Eskin, Boundary Value Problems for Elliptic Pseudodifferential Equations (AMS, Providence, 1981)

4. S. Hofmann, M. Mitrea, M. Taylor, Singular integrals and elliptic boundary problems on regular semmes-Kenig-Toro domains. Int. Math. Res. Not. 14, 2567-2865 (2010)

5. R.B. Melrose, Pseudodifferential operators, corners and singular limits. In Proceeding of the International Congress of Mathematicians, 21-29 August 1990, Kyoto, Japan, ed. by Satake, vol. I (Springer, Tokyo, 1991), pp. 217-234

6. S. Moroianu, V. Nistor, Index and homology of pseudodifferential operators on manifolds with boundary. In Perspectives in Operator Algebras and Mathematical Physics. Theta Series in Advanced Mathematics, vol. 8 (Theta, Bucharest, 2008), pp. 123-148

7. V. Nistor, Analysis on singular spaces: lie manifolds and operator algebras. J. Geom. Phys. 105, 75-101 (2016)

8. M. Ruzhansky, N. Tokmagambetov, Nonharmonic analysis of boundary value problems. Int. Math. Res. Not. 12, 3548-3615 (2016)

9. I.B. Simonenko, A local method in the theory of translation invariant operators and their envelopings (in Russian) (CVVR Publishing, Rostov on Don, 2007)

10. V.B. Vasil'ev, Regularization of multidimensional singular integral equations in non-smooth domains. Trans. Mosc. Math. Soc. 59, 65-93 (1998)

11. V.B. Vasil'ev, Wave Factorization of Elliptic Symbols: Theory and Applications (Kluwer Academic Publishers, Dordrecht, 2000)

12. V.B. Vasilev, Pseudodifferential equations in cones with conjugate points on the boundary. Differ. Equ. 51, 1113-1125 (2015)

13. V.B. Vasilyev, Asymptotical analysis of singularities for pseudo differential equations in canonical non-smooth domains. In Integral Methods in Science and Engineering, ed. by C. Constanda, P. Harris (Birkhäuser, Boston, 2011), pp. 379-390

14. V.B. Vasilyev, Pseudo differential equations on manifolds with non-smooth boundaries. In Differential and Difference Equations and Applications, ed. by S. Pinelas et al. Springer Proceedings in Mathematics \& Statistics, vol. 47 (Birkhäuser, Basel, 2013), pp. 625-637

15. V.B. Vasilyev, New constructions in the theory of elliptic boundary value problems. In Integral Methods in Science and Engineering, ed. by C. Constanda, A. Kirsch (Birkhäuser, New York, 2015), pp. 629-641

16. V.S. Vladimirov, Methods of the Theory of Functions of Many Complex Variables (Dover Publications, Mineola, 2007) 